\title{
The sympathetic nervous system stimulates anti-inflammatory B cells in collagen-type II-induced arthritis
}

\author{
Georg Pongratz, Madlen Melzer, Rainer H Straub
}

Laboratory of Experimental Rheumatology and Neuroendocrine Immunology, Department of Internal Medicine I, University Hospital Regensburg, Regensburg, Germany

\section{Correspondence to} Georg Pongratz, Laboratory of Experimental Rheumatology and Neuroendocrine Immunology, Department of Internal Medicine I, University Hospital Regensburg, 93042 Regensburg, Germany; georg.pongratz@klinik. uni-regensburg.de

Received 23 February 2011 Accepted 14 August 2011 Published Online First 27 September 2011

\begin{abstract}
Background As previously shown, the sympathetic nervous system (SNS) shows proinflammatory activity during initiation of arthritis but is anti-inflammatory in established collagen-induced arthritis (CIA). Interleukin 10 (IL-10)-producing B cells suppress arthritis and are a potential target of the SNS because (1) B cells express functional $\beta_{2}$-adrenoceptors ( $\beta_{2}$ ARs) and (2) IL-10, at least in monocytes/macrophages, is regulated in a cAMP/ PKA/CREB-dependent manner.

Objective To test the hypothesis that anti-inflammatory effects of the SNS in CIA are mediated by stimulating IL-10-producing anti-inflammatory B cells.

Methods Collagen-induced arthritis in DBA/1 mice, sympathectomy, adoptive B cell transfer, in vitro B cell culture, and assessment of B cell IL-10 production.

Results and conclusion Mice treated with B cells from SNS-intact mice showed less severe arthritis than mice treated with B cells from sympathectomised mice. This anti-inflammatory action of B cells from SNS-intact mice correlated with increased IL-10 produced by B cells, which was mediated by norepinephrine (NE), in a $\beta_{2} A R$, PKA-dependent manner. However, an NE-mediated increase in IL-10 was seen only in B cells from immunised but not naive mice, explaining in part the anti-

inflammatory properties of the SNS in the late phase of arthritis. Finally, animals treated with B cells isolated from immunised mice and activated in vitro in the presence of a $\beta_{2} A R$ stimulus showed a decrease in arthritis severity in comparison with controls, an approach that might be used for future cellular treatment strategies.
\end{abstract}

\section{INTRODUCTION}

The depletion of CD20+ B cells as a treatment option for some rheumatic disorders-for example, rheumatoid arthritis, has raised again the proposition that $\mathrm{B}$ cells have a pathogenetic role in these disorders. However, depletion of all CD20+ cells is an unspecific approach and risks the occurrence of serious side effects such as infection. The optimal treatment for $B$ cells would be specific depletion of autoreactive B cells, on the one hand, and promotion of anti-inflammatory $\mathrm{B}$ cells, on the other. The mechanisms by which antiinflammatory B cells suppress chronic inflammation in arthritis are not completely understood. However, one hallmark of anti-arthritic regulatory B cells is their ability to produce interleukin 10 (IL-10). ${ }^{1}$

IL-10 is an anti-inflammatory cytokine, which suppresses antigen presentation by antigen-presenting cells, downregulates major histocompatibility complex II molecules and inhibits the expression of co-stimulatory molecules, preventing sufficient costimulation of antigen-specific T cells. Additionally, IL-10 directly suppresses the production of proinflammatory cytokines, chemokines and matrix metalloproteinases in macrophages (reviewed by Mosser and Zhang ${ }^{2}$ ), which explains its anti-inflammatory potential in arthritis. The overall effect of IL-10 on the whole B-cell population is stimulatory rather than immunosuppressive because IL-10 leads to prolonged survival, augments co-stimulation and induces class switch recombination. ${ }^{2}$ Nevertheless, a subpopulation of IL-10-producing B cells, so called CD1 $d^{\text {high }}$ CD5 ${ }^{\text {high }}$ B10 cells, ${ }^{3}$ clearly shows antiinflammatory properties in arthritis. ${ }^{14}$

The human and murine promoter of the IL-10 gene contains several CREB-responsive elements. ${ }^{5} 6$ Thus, it is not surprising that $\mathrm{IL}-10$ production can be regulated through PKA/CREB-dependent mechanisms, which have been shown in THP-1 cells and macrophages. ${ }^{5}$ Activity of PKA is increased by cAMP, which is generated, following the stimulation of Gs protein coupled receptors, such as the $\beta_{2}$-adrenoceptor ( $\left.\beta_{2} \mathrm{AR}\right)$; a functional $\beta_{2} \mathrm{AR}$ has also been shown to be expressed on $B$ cells. ${ }^{89}$ Additionally, we reported in collagen type II-induced arthritis (CIA) that the sympathetic nervous system (SNS) with its main neurotransmitter norepinephrine (NE) has a role in regulating IL-10 in arthritis and has a profound effect on arthritis severity. ${ }^{10}$ Furthermore, it has been shown that the SNS plays a proinflammatory role in the early phase and an anti-inflammatory role in the late phase of the disease. ${ }^{1011}$ The proinflammatory effect of the SNS in the early phase of the disease can be mediated via increasing proinflammatory cytokines, such as interferon $\gamma$ produced by $\mathrm{T}$ cells. ${ }^{12}$ Additionally, it has been reported that FOXP3-negative CD4+CD25+ $T$ cells also contribute to the proinflammatory effect of the SNS in the early phase of CIA. ${ }^{11}$ However, the anti-inflammatory action of the SNS in the late phase of CIA has not yet been elucidated.

In this study, we tested the hypothesis that anti-inflammatory effects of the SNS in CIA are mediated by stimulating IL-10 producing antiinflammatory B cells.

\section{MATERIAL AND METHODS Animals, induction of arthritis and arthritis scoring}

Male DBA/1 mice 6-8 weeks old (Elevage Janvier, Le Genest St Isle, France) were housed in cages and 
fed standard laboratory chow and water ad libitum. There was a 12 h light-dark cycle. Experiments were conducted according to institutional and governmental regulations for animal use (Government of the Oberpfalz AZ 54-2531.1-24/06).

Experimental arthritis was induced in DBA/1 mice, as previously described. ${ }^{10}$ On day 0 , mice were immunised intradermally at the base of the tail with $100 \mu \mathrm{g}$ of bovine type II collagen (CII; Chondrex, Redmond, Washington, USA) emulsified in an equal volume of complete Freund's adjuvant (CFA; Sigma-Aldrich Chemie, München, Germany). A booster immunisation (100 $\mu \mathrm{g}$ CII in CFA) was administered on day 21 as indicated. Clinical scoring points were assigned to each limb to assess arthritis as described previously. ${ }^{13}$ A score of 0 (no swelling), 1 (light swelling) or 2 (strong swelling) was determined for four toes at each paw, four paws and ankle/wrist joints. The maximum score for each mouse was 48 points. Arthritis scoring was performed in a blinded manner, by the same technical assistant, always at the same time of the day (11:00-12:00).

\section{Adoptive transfer experiments}

Three groups of mice were immunised as described above (control donor mice, sympathectomised donor mice and sympathectomised recipient mice, figure $1 \mathrm{~A}$ ). On day 28 , donor mice were killed by cervical dislocation, spleens were removed and immediately put into ice-cold phosphate-buffered saline (PBS). Splenectomy was performed in recipient mice at day 28 to remove the majority of the intrinsic B-cell pool. After preparing single-cell suspensions of donor splenocytes, B cells were isolated by magnetic bead cell sorting using mouse anti-B220 magnetic beads (MACS MicroBeads; Miltenyi Biotec, Bergisch Gladbach, Germany). After isolation, $>95 \%$ of the cells were CD19+/CD20+ B cells as assessed by fluorescence activated cell sorter (FACS) analysis. Donor B cells $\left(5 \times 10^{6} / 200 \mu \mathrm{l}\right.$ PBS) were then injected into the tail vein of recipient mice (day 28 post-immunisation (p.i.)). One group received $200 \mu \mathrm{PBS}$ as treatment control (figure 1A). After transfer, clinical scores were obtained every 2-3 days until the end of experiment (day 63). After sacrificing the mice, heart blood was obtained and anti-CII antibody titres were determined in serum using a commercially available ELISA kit (MD Biosciences GmbH, Zürich, Switzerland).

\section{Culture of splenocytes and B cells}

Spleens were removed between 11:00 and 12:00. Single-cell suspensions were obtained by passing spleens through a nylon mesh. For splenocyte cultures, $5 \times 10^{5}$ cells $/ 200 \mu \mathrm{l}$ were cultured in complete RPMI medium (cRPMI: RPMI 1640, 25 mM HEPES, pH 7.4, $5 \%$ fetal calf serum, $30 \mu \mathrm{M}$ mercaptoethanol, $0.57 \mathrm{mM}$ ascorbic acid, $100 \mathrm{U} / \mathrm{ml}$ penicillin, $100 \mu \mathrm{g} / \mathrm{ml}$ streptomycin (all from Sigma, Munich, Germany)) in 96-well plates and stimulated with anti-CD40 (clone: FGK 45.5, Miltenyi Biotec, Bergisch Gladbach, Germany, $5 \mu \mathrm{g} / \mathrm{ml}$ ) and bovine CII (Chondrex, Redmond, Washington, USA, $50 \mu \mathrm{g} / \mathrm{ml})$ in the presence or absence of NE $\left(10^{-6}\right.$ M, Sigma) for 4-5 days. For B-cell cultures, cells were isolated using magnetic bead cell sorting by depleting CD43-, CD4- and Ter119+ cells from whole splenocyte suspensions (B-cell isolation kit, Miltenyi Biotec, Germany). After isolation, $>96 \%$ of the cells were CD19/CD20 positive B cells. Cells were resuspended in cRPMI containing anti-CD40 (5 $\mu \mathrm{g} / \mathrm{ml}$, clone: FGK 45.5, Milteyi Biotec, Bergisch Gladbach, Germany) and functional grade IL-4 ( $1 \mathrm{ng} / \mathrm{ml}$, eBioscience, Frankfurt, Germany) and cultured at a density of $2 \times 10^{6}$ cells $/ \mathrm{ml}$ in 24 -well plates for 4-5 days in the presence or absence of NE, the $\beta_{2} \mathrm{AR}$ specific agonist terbutaline, the protein kinase $A(P K A)$ inhibitor $\mathrm{H} 89$, or the specific $\beta_{2} A R$ antagonist nadolol at indicated concentrations. For experiments using H89 or nadolol, cells were incubated for $30 \mathrm{~min}$ before adding NE or terbutaline, respectively.

\section{Treatment of mice with early arthritis with cultured B cells}

Splenic B cells were obtained from immunised mice at day 21 after immunisation and resuspended in cRPMI containing antiCD40/IL-4 as described above. B cells were cultured for 5 days in 12 -well plates at of $2 \times 10^{6} / \mathrm{ml}$ in the presence or absence of $\mathrm{NE}$, or terbutaline, or terbutaline + nadolol as indicated. After 5 days, cells were counted, washed five times and resuspended in $\mathrm{PBS}$ at $2 \times 10^{6}$ live B cells $/ 100 \mu \mathrm{l}$. Immunised mice beginning to show signs of synovitis at day 18 were treated with $2 \times 10^{6} \mathrm{~B}$ cells intravenously and the arthritis score was recorded thereafter.

\section{Restimulation and assessment of IL-10 production}

Restimulation of cultured cells and assessment of intracellular IL-10 was performed in accordance with methods published elsewhere. ${ }^{13}$ Briefly, after 4-5 days of culture cells were stimulated with phorbol myristate actetate (PMA; $50 \mathrm{ng} / \mathrm{ml}$; Sigma), ionomycin (500 ng/ml; Sigma) and lipopolysaccharides (LPS, $10 \mu \mathrm{g} / \mathrm{ml}$ ), as indicated. For IL-10 protein detection, cell-free supernatant was analysed using a BD OptEIA mouse IL-10 ELISA kit (BD Bioscience, Heidelberg, Germany). For intracellular FACS analysis, brefeldin A (10 $\mu \mathrm{g} / \mathrm{ml}$; Sigma) was added together with PMA and ionomycin. Cells were stained with fluorescein isothiocyanate-anti-mouse CD19 (clone: MB 19-1, eBioscience) for $15 \mathrm{~min}$ on ice. Then cells were fixed (2\% paraformaldehyde, $20 \mathrm{~min}$ at $4^{\circ} \mathrm{C}$ ), permeabilised using Cytofix/ Cytoperm (BD-Pharmingen, Heidelberg, Germany) and stained with phycoerythrin-IL-10 monoclonal antibody (clone: JES516E3; eBioscience) or respective isotype control for $25 \mathrm{~min}$ on ice. FACS analysis was performed on a FACS Calibur (Becton Dickinson Immunocytometry Systems, San Jose, California, USA). Data were analysed with FlowJo Software (V.7.2.4.; Tree Star Inc, Ashland, Oregon, USA) or WinMDI2.8 (http://facs. scripps.edu/software.html), respectively.

\section{Sympathectomy}

Sympathectomy was performed using saporin-coupled antidopamine- $\beta$-hydroxylase antibodies or saporin-coupled control antibodies (Advanced Targeting Systems, San Diego, California, USA $)^{10} 11$ by intraperitoneal injection ( $5 \mu$ g per animal in PBS) 7 days before immunisation. The NE content in the spleen was reduced by $78 \%$ at the time of immunisation, demonstrating the efficacy of sympathectomy.

\section{Statistical analysis}

The non-parametric Mann-Whitney test was used to compare two groups and analysis of variance with post hoc tests was used to compare multiple groups. All analyses were conducted using Sigma Plot for Windows (version 11; Systat software Inc.). p Values $<0.05$ were considered significant.

\section{RESULTS \\ The SNS ameliorates CIA by promoting anti-inflammatory $B$ cells in vivo}

To test the hypothesis that the SNS promotes the development of anti-inflammatory B cells in CIA, B cells were adoptively transferred as described above and as shown in figure1A. In arthritic mice treated with B cells from SNS-intact mice arthritis 

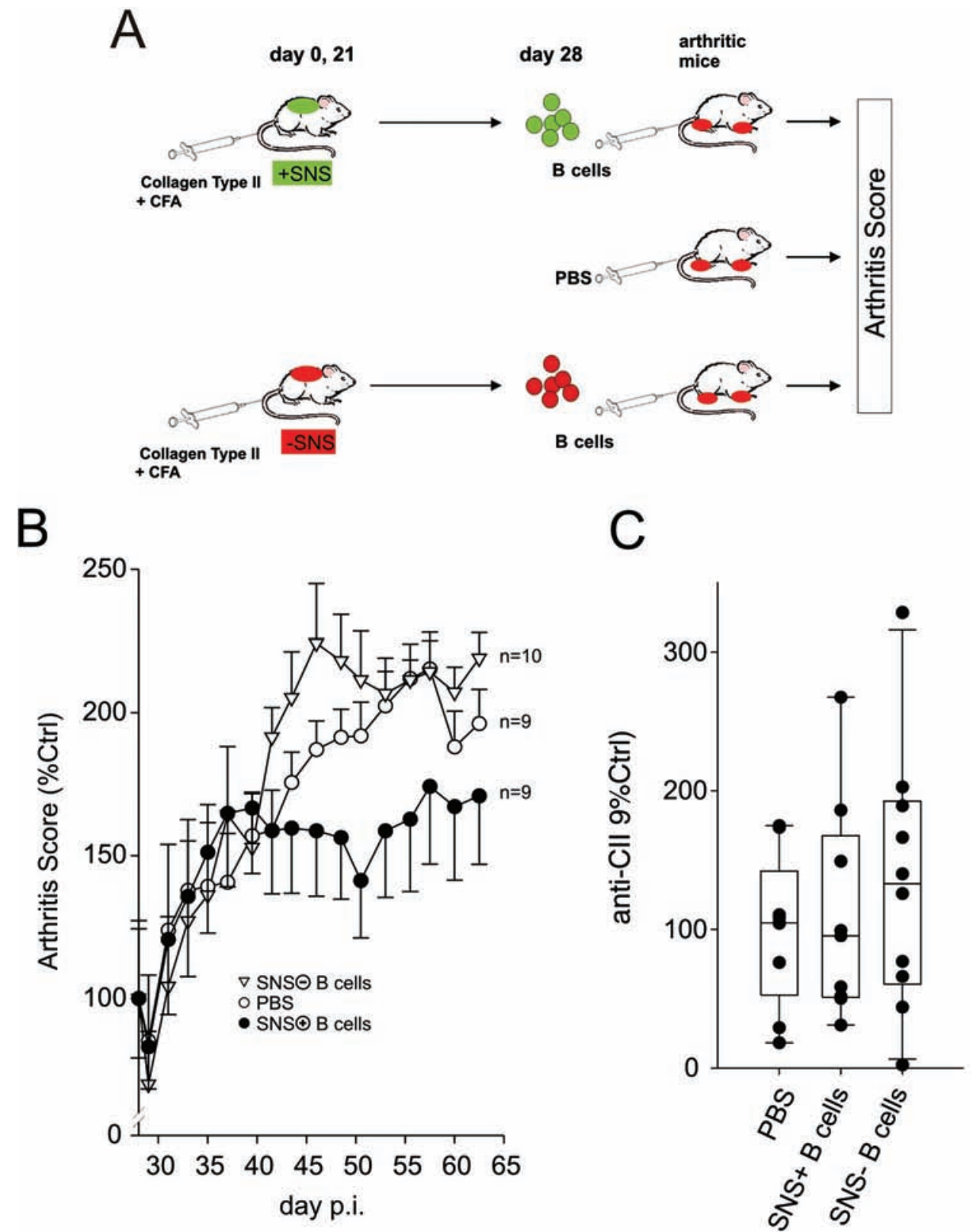

Figure 1 B cells obtained from sympathetic nervous system (SNS)-intact mice show regulatory potential in collagen type II-induced arthritis. (A) Experimental design: DBA1/J mice were depleted of the sympathetic nervous system by saporin (SAP)-conjugated anti-dopamine $\beta$-hydroxylase antibodies (depleted sympathetic nervous system (SNS $\Theta)$ ), or treated with SAP-conjugated isotype control antibodies (SNS $\oplus$ ) 7 days before immunisation with bovine collagen type II in complete Freund's adjuvant. On day 28, splenectomy was performed, B cells were isolated and SNS $\Theta$ arthritic mice were treated with B cells from either SNS $\Theta$, or SNS $\oplus$ donors, respectively. One group was treated with phosphate-buffered saline (PBS) only. (B) Transfer of SNS $\oplus$ B cells stops progression of collagen-induced arthritis. Arthritis score was determined by a blinded observer during the indicated time period post-immunisation (days p.i.). A general linear statistical model (two-way analysis of variance with Holm-Sidak post hoc analysis) showed between-group differences for mice treated with SNS $\oplus$ B cells versus mice treated with PBS $(p=0.008)$ and mice treated with SNS $\Theta$ B cells $(p<0.001)$, respectively. No significant group difference was found between mice treated with PBS and mice treated with SNS $\Theta$ B cells $(p=0.09)$. The average score of two independent experiments (\% Ctrl) is shown. $n=$ total number of mice. Error bars represent the SEM. (C) Serum level of anti-collagen type II antibodies (anti-CII) at the last day of the experiment as determined by ELISA.

progression stopped about 14 days after treatment (figure1B), whereas treatment with B cells obtained from SNS-depleted animals resulted in a short-term aggravation of the disease as compared with the PBS-treated control group (figure $1 \mathrm{~B}$ ). This result supports the hypothesis that the SNS promotes development of $B$ cells that show anti-inflammatory properties in the CIA mouse model. The effect was not mediated by a change in antiCII antibody titres at day 63. FACS analysis also showed that the percentage of $\mathrm{B}$ cells in the spleen and in peripheral blood was not influenced by sympathectomy (data not shown).

\section{The SNS increases IL-10 produced by B cells in vivo}

B cells producing IL-10 have an anti-inflammatory action in CIA, and the regulatory regions of the IL-10 gene contain cAMPresponsive elements, ${ }^{16}$ rendering IL-10-producing $B$ cells a possible target for the SNS. To determine if the SNS changes the 
A

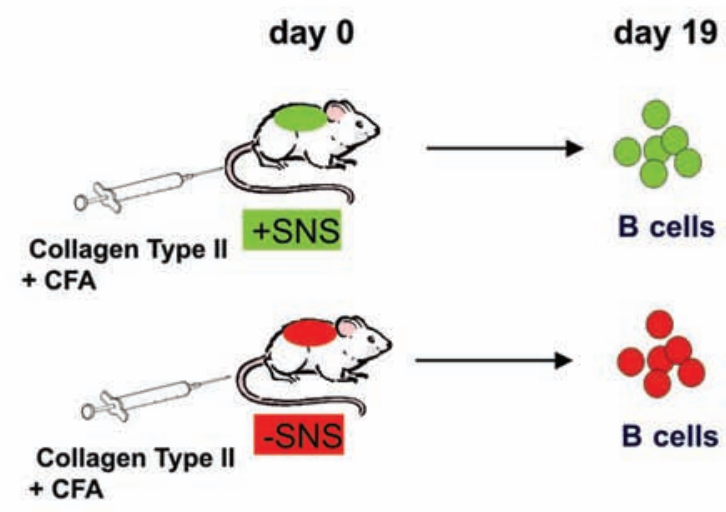

B

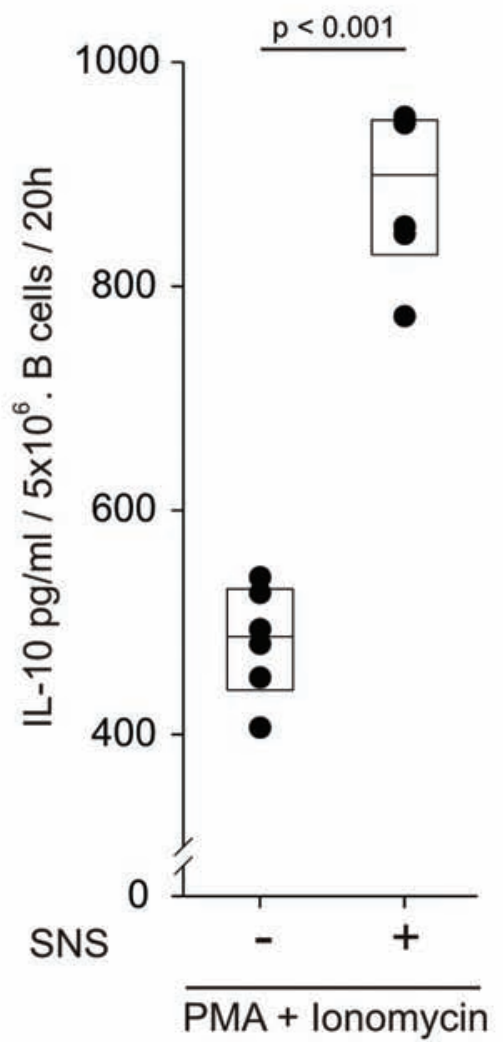

Figure 2 Interleukin (IL)-10 produced by splenic B cells is increased via the sympathetic nervous system in vivo following immunisation. (A) Experimental design. DBA/1J mice were treated with saporincoupled anti- $\beta$ hydroxylase antibodies $(n=6)$ or saporin-coupled isotype control antibodies $(n=6) 7$ days before immunisation with bovine collagen type II in complete Freund's adjuvant (CFA, day 0). (B) At day $19,5 \times 10^{6}$ splenic $B$ cells were stimulated with phorbol myristate actetate $(50 \mathrm{ng} / \mathrm{ml})$ and ionomycin $(500 \mathrm{ng} / \mathrm{ml})$ for $20 \mathrm{~h}$ and IL-10 was determined in cell-free supernatants by ELISA. p Value was determined by Mann-Whitney test. Results are presented as box plots, horizontal lines represent the median value. SNS, sympathetic nervous system.

ability of B cells to produce IL-10 in vivo, splenic B cells obtained from immunised and non-immunised mice at day 19 p.i. from SNS-depleted and SNS-intact mice were compared (figure 2A). $B$ cells obtained from SNS-depleted mice produced significantly less IL-10 than the same number of B cells from SNS-intact mice (figure 2B). When B cells were isolated from non-immunised animals sympathectomy did not influence the amount of IL-10 produced (data not shown). These result suggest that one function of the SNS in vivo is to promote IL-10-producing B cells during activation after immunisation with CII and CFA.

\section{Norepinephrine differentially regulates IL-10 produced by B cells from naive or antigen-experienced spleens}

Owing to earlier findings that the SNS shows proinflammatory properties during the early phase and anti-inflammatory properties during the late phases of CIA, ${ }^{10}$ it was determined if NE, the main SNS neurotransmitter, differentially regulates IL-10 produced by cultured naïve splenocytes as compared with splenocytes obtained from antigen-experienced mice. Antigen-experienced splenocytes cultured for 4-5 days in the presence of anti-CD40, CII and NE showed increased IL-10 production after restimulation with PMA, ionomycin and LPS, as compared with cells in the absence of NE (figure 3A). FACS analysis showed that an increase in intracellular IL-10 in lymphocytes was mainly seen in CD19+ B cells (figure 3B). However, this NE-induced increase in IL-10 was not seen when naïve-splenocytes were used in a similar experiment (figure $3 \mathrm{C}$ ), indicating that immunisation before stimulation with NE renders some IL-10-producing, antigen-experienced splenocytes-for example, B cells, responsive to this neurotransmitter.

\section{Norepinephrine acts directly on B cells to increase IL-10 production}

To determine whether the increase in IL-10-producing B cells, after activation of splenocytes in the presence of NE is dependent on cells other than B cells in the culture, we isolated B cells from naive and antigen-experienced spleens and activated them with anti-CD40 and IL-4 in the absence (Ctrl) or presence of NE for 5 days and analysed IL-10+/CD19+ cells by FACS (figure $4 \mathrm{~A}$ ). The results are expressed as a percentage of control (\% Ctrl, anti-CD40/IL-4 only). The absolute values for the percentage of IL-10+/CD19+ B cells from naïve mice $(3.1 \% \times 1.2)$ and antigenexperienced mice $(2.6 \% \times 1.3)$ were not significantly different $(p=0.953)$. As shown in figure $4 \mathrm{~A}, \mathrm{NE}$ in a concentration-dependent manner increases IL-10+/CD19+ B cells when isolated from spleens of mice immunised with CII/CFA (day p.i. 35-65). The increase was significant at concentrations of $10^{-8}$ to $10^{-6} \mathrm{~mol} / \mathrm{l}$ (figure 4A, solid circles). However, when $\mathrm{B}$ cells from naive mice were used, the population of IL-10+ B cells did not change in the presence of NE (figure 4A, open circles). These results show that NE directly acts on B cells to increase IL-10+ cells, but only when the cells were taken from antigen-experienced spleens.

\section{Norepinephrine increases IL-10 produced by

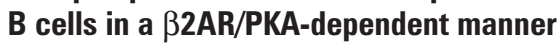

Since it is known that $B$ cells express functional $\beta_{2} A R$, we next determined if specific stimulation of this receptor with terbutaline could mimic the effect of NE. Terbutaline increased IL-10 protein produced by B cells (isolated at day 35-40 p.i.) in a concentration-dependent manner (figure 4B). Additionally, the use of a specific PKA inhibitor, H89, showed that the terbutaline-induced increase in IL-10 was mediated through a PKAdependent pathway (figure 4C). The addition of $\mathrm{H} 89$ prevented the increase of IL-10, and also reversed the terbutaline effect, resulting in decreased IL-10 production. This result suggests a second signalling pathway downstream of the $\beta_{2} A R$, which leads to a decrease in IL-10 production upon stimulation of the receptor. Taken together, these results suggest that the increase 
A

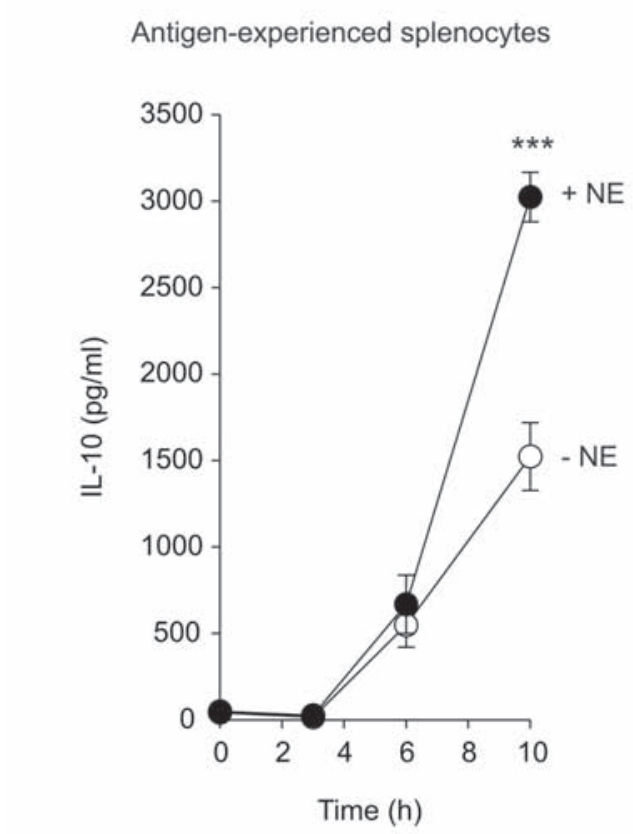

C

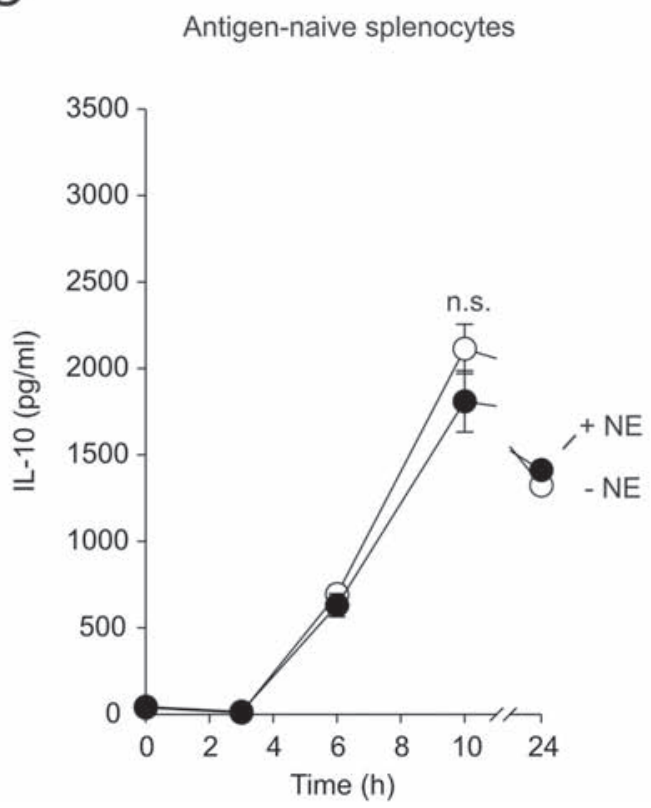

B

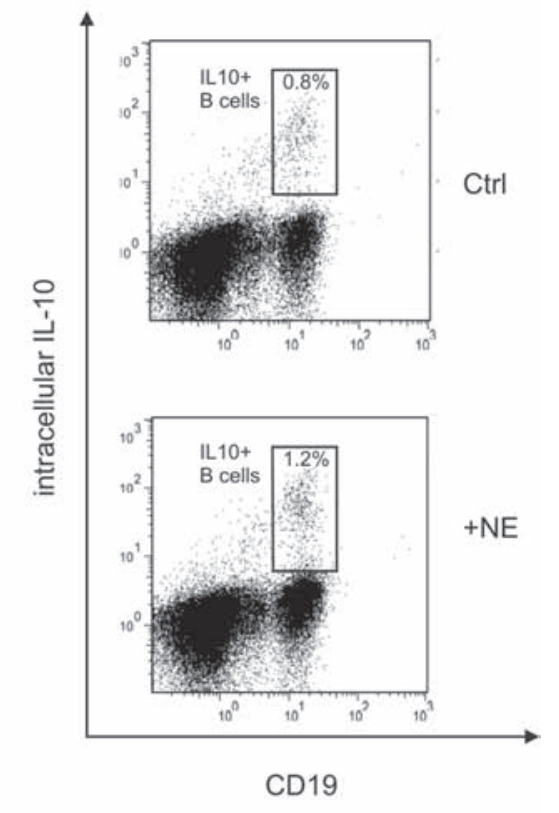

Figure 3 Norepinephrine differentially regulates interleukin (IL)-10 produced by antigen experienced or naïve splenocytes, respectively. Splenocytes from mice immunised with collagen type II and complete Freund's adjuvant $(A,>$ day 60 post-immunisation) or naïve spleen cells from DBA1/J mice $(C)$ were activated with anti-CD40 and bovine collagen type II in the presence (+NE) or absence (-NE) of norepinephrine $\left(10^{-6} \mathrm{M}\right)$. After $4-5$ days, cells were washed and stimulated with phorbol myristate actetate, ionomycin and lipopolysaccharide, and IL-10 protein concentration was determined by ELISA at indicated time points. ${ }^{* *} p<0.001$ was determined by Mann-Whitney $U$ test. n.s., not significant. Results of four independent experiments with four replicates each are shown. (B) Representative FACS analysis of stimulated splenocytes after culture in the absence (Ctrl) or presence $(+\mathrm{NE})$ of norepinephrine $10^{-6} \mathrm{M}$. Analysis of expression of intracellular IL-10 and CD19 as indicated. The number in the rectangular region indicates the percentage of IL10+/CD19+ cells.

in IL-10 produced by anti-CD40/IL-4-activated B cells isolated from antigen-experienced spleens is mediated via the $\beta_{2} \mathrm{AR}$ in a PKA-dependent manner.

\section{Treatment of CIA with $\beta 2 A R-s t i m u l a t e d$ $B$ cells isolated from antigen-experienced mice ameliorates disease progression}

To show that the increase in IL-10+B cells after $\beta_{2}$ AR stimulation of $B$ cells from antigen-experienced mice in vitro is of relevance in vivo, arthritic mice were treated with B cells activated in the absence or presence of $\mathrm{NE}$, terbutaline, or terbutaline + nadolol, respectively (figure 5A). Mice treated with anti-CD40/ IL-4-activated B cells (B-cell control) or PBS, respectively, showed the expected increase of arthritis severity over time (figure $5 \mathrm{~B}$ ). There was a trend towards a faster increase of arthritis scores in the B-cell control group as compared with PBS control in the first 10 days after treatment $(p=0.074)$, but no overall significant difference $(p=0.099)$. On the other hand, mice receiving $B$ cells activated in the presence of either terbutaline or NE showed significantly reduced arthritis severity as compared with controls 

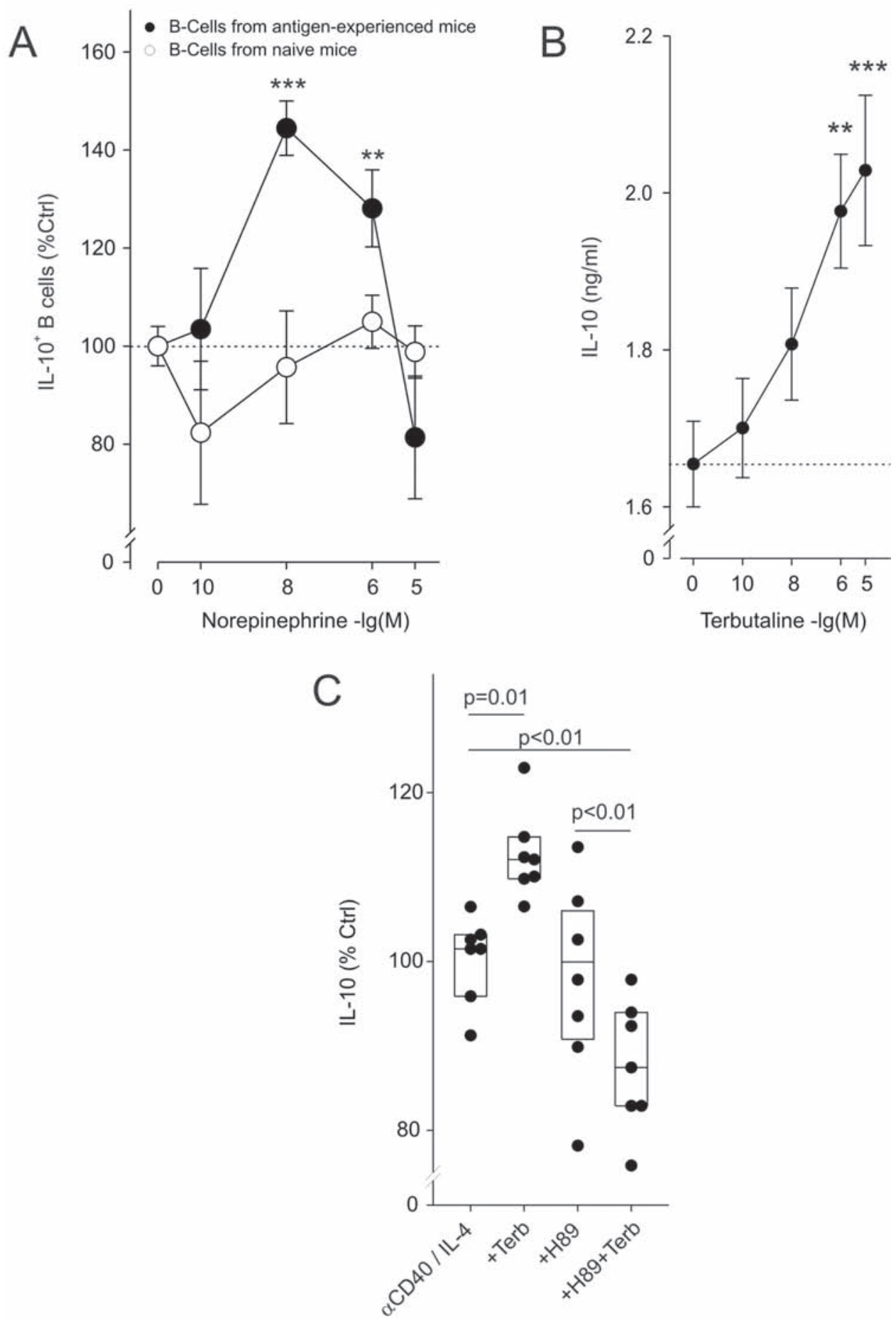

Figure 4 Norepinephrine increases interleukin (IL)-10-producing splenic $B$ cells via stimulation of $\beta 2$-adrendergic receptors in a concentrationdependent and protein kinase $A$-dependent manner. Splenic $B$ cells from antigen-experienced mice $(A$, solid circles) and naïve mice $(A$, open circles) were cultured with anti-CD40 and IL-4 in the absence or presence of norepinephrine (A) or terbutaline (B) at indicated concentrations. (C) Terbutaline (Terb) was used at $10^{-6} \mathrm{M}, \mathrm{H} 89$ at $1 \mu \mathrm{g} / \mathrm{ml}$. After $4-5$ days of culture, B cells were stimulated with phorbol myristate actetate, ionomycin and lipopolysaccharide in the presence $(A)$ or absence $(B, C)$ of breveldin $A$ for $8-10 \mathrm{~h}$. The percentage of IL10+/CD19+ B cells or IL-10 protein concentration was determined by FACS analysis (A) or ELISA (B, C), respectively. Mean values expressed as percentage of control (anti-CD40/IL-4 only, \% Ctrl) of six independent experiments ( $A$, closed circles) and eight independent experiments ( $A$, open circles) are shown. $(B, C)$ Mean values for three independent experiments $(B)$ and two independent experiments $(C)$ are shown. $p$ Values were determined by analysis of variance with Holm-Sidak $(A, C)$ and Bonferroni (B) post hoc tests, respectively. Error bars represent the SEM. ${ }^{* * *} \mathrm{p}<0.001 ;{ }^{* *} \mathrm{p}<0.01$.

(figure 5). Additionally, mice treated with B cells activated in the presence of terbutaline and nadolol did not differ as compared with the control groups $(p=0.074)$. Taken together the results suggest that a $\beta_{2} A R$ stimulus during activation of $B$ cells from antigen-experienced mice in vitro increases the anti-inflammatory activity of these cells in vivo, by increasing IL-10+ B cells. 
A

day 0

day 21-26

arthritic mice

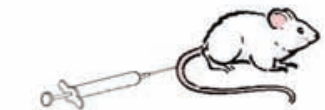

Collagen Type II

+ CFA

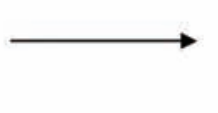

splenic B cell culture

1: anti-CD40/IL-4

2: + Norepinephrine

3: + Terbutaline

4: + Terbutaline + Nadolol

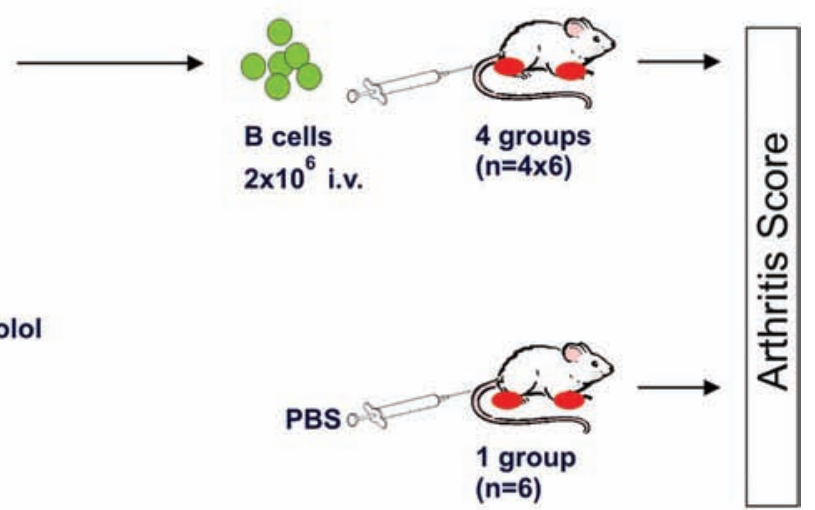

B

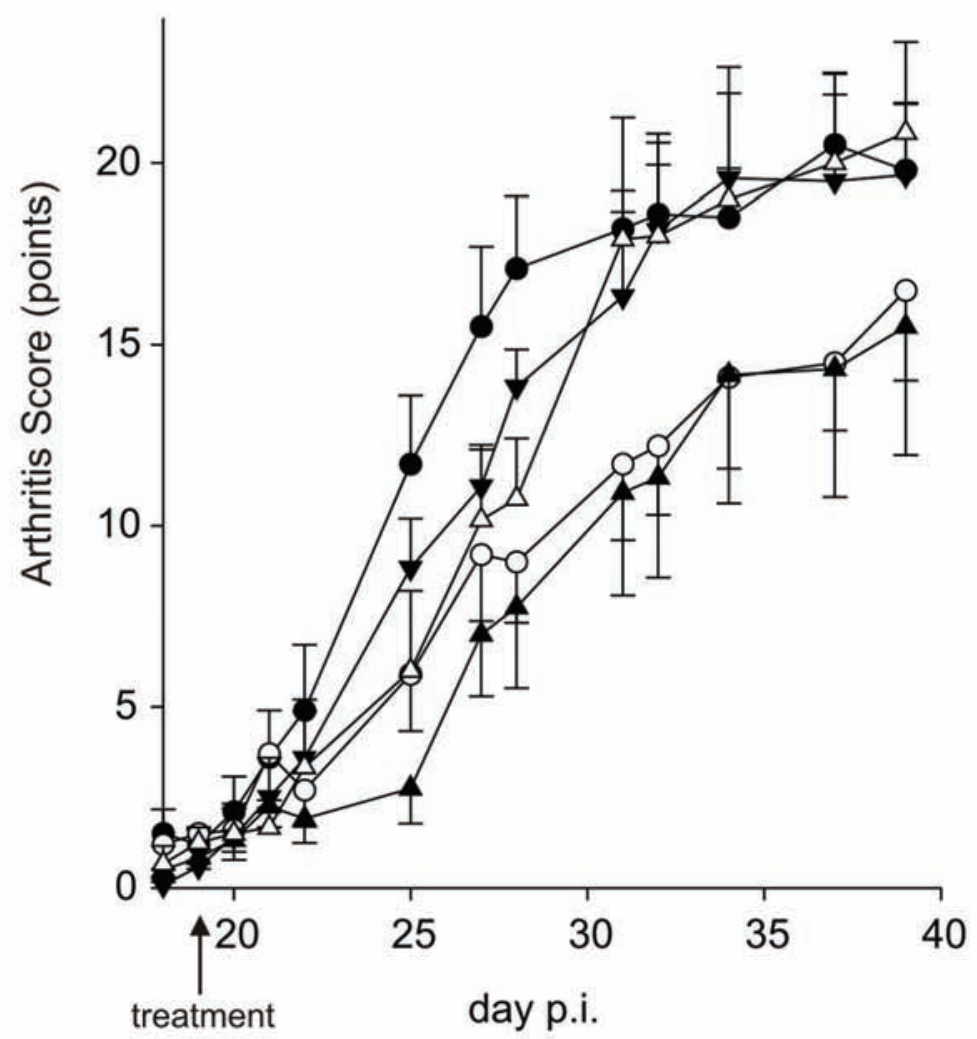

- anti-CD40/IL-4

$-\mathrm{O}-$ + Norepinephrine

- $\_$+ Terbutaline

$\rightarrow-+$ Terbutaline + Nadolol

$\triangle-+$ PBS

Figure 5 Treatment of collagen-induced arthritis with $B$ cells activated in vitro in the presence of a $\beta_{2}$-adrenoceptor $\left(\beta_{2} A R\right)$ agonist ameliorates disease progression. (A) Experimental design: five mice were immunised with bovine collagen type II (CII) and complete Freund's adjuvant (CFA). At day 21 post immunisation (p.i.) naïve splenic B cells were isolated and activated for 5 days with anti-CD40/interleukin (IL)-4 in the presence or absence of norepinephrine $\left(10^{-6} \mathrm{M}\right)$, terbutaline $\left(10^{-6} \mathrm{M}\right)$, or terbutaline $\left(10^{-6} \mathrm{M}\right)+$ nadolol $\left(10^{-5} \mathrm{M}\right)$. B cells were then vigorously washed and resuspended in phosphate-buffered saline (PBS). Six mice with collagen-induced arthritis per group (anti-CD40/IL-40, + norepinephrine, + terbutaline, + terbutaline+ nadolol) were injected intravenously with $2 \times 10^{6}$ B cells per mouse or with PBS only at day 18 p.i. (B) Transfer of $\beta_{2} A R$-treated B cells significantly slowed arthritis progression. Arthritis score was determined by a blinded observer during the indicated time period post immunisation (days p.i.). A general linear statistical model (two-way analysis of variance with Student-Newman-Keuls post hoc analysis) showed between-group differences for mice treated with B cells activated in the presence of norepinephrine or terbutaline, respectively, as compared with mice treated with PBS, or B cells activated with anti-CD40/IL-4 only, or B cells activated in the presence of nadolol + terbutaline ( $p<0.001$ for all comparisons). No significant group difference was found between mice treated with PBS and mice treated with B cells activated in the presence of nadolol + terbutaline or B cells activated with anti-CD40/IL-4 only. Symbols represent the group means, the error bars represent the SEM.

\section{DISCUSSION}

Recent studies using the CIA model system show, that the SNS has a dual role with respect to disease severity, with proinflammatory activity in the early phase and an anti-inflammatory influence in the late phase of arthritis. ${ }^{10} 11$ Our results in this study suggest that the anti-inflammatory activity of the SNS in the late phase of arthritis is, at least in part, mediated by increasing the anti-inflammatory properties of $\mathrm{B}$ cells. We propose 
that in the late phase of CIA a sympathetic stimulus increases the percentage of IL-10-producing, anti-inflammatory B cells, which can slow down arthritis progression. ${ }^{1}$ Our results do not exclude the possibility that the SNS also influences other cells-for example, monocytes/macrophages, which have been shown to increase IL-10 production after stimulation of $\beta_{2} A R$ in the human system. ${ }^{5714}$ However, transferring splenic $B$ cells in vitro, activated in the presence of a $\beta_{2} A R$ stimulus, is sufficient to significantly slow arthritis progression, showing the important role of the SNS and B cells, respectively, in modulating disease severity in arthritis.

A necessary prerequisite for the SNS to increase IL-10-producing B cells was prior immunisation with CII and CFA, which underlines the importance of this mechanism after initiation of arthritis (late phase). The need for prior immunisation indicates that a proinflammatory milieu in the spleen is important to render naive $\mathrm{B}$ cells responsive for a $\beta_{2} \mathrm{AR}$-induced increase in IL-10. The reason for this is unclear. However, one possibility might be an increase in $\beta_{2} \mathrm{AR}$ signalling capacity by decreasing G-protein-coupled receptor kinase (GRK)-2, which was reported to be decreased in peripheral blood mononuclear cells from patients with rheumatoid arthritis and upon antigen exposure in $\mathrm{B}$ and $\mathrm{T}$ cells in a rat model of adjuvant arthritis. ${ }^{15} 16$ This leads to the hypothesis that an immune challenge decreases GRK-2 in naïve B cells, resulting in less $\beta_{2} \mathrm{AR}$ phosphorylation, which slows internalisation and desensitisation of $\beta_{2}$ ARs. This results in increased and prolonged $\beta_{2}$ AR signalling, which might cross a certain threshold to initiate PKA-dependent IL-10 production in B cells.

The importance of IL-10 in modulating arthritis severity is underlined by several correlative studies showing a link between genetic variation at the IL-10 gene locus and severity of arthritis, ${ }^{17} 18$ but human studies treating arthritis with systemic IL-10 showed almost no effect (reviewed by Asadullah et a ${ }^{19}$ ). However, a small number of antigen-specific $T$ cells transfected to produce IL-10 were capable of suppressing inflammation in proteoglycan-induced arthritis, ${ }^{20}$ probably by cell-cell interaction at the site of inflammation without an increase in systemic IL-10. This fits together with our results, which show no change in IL-10 serum levels upon transfer of $\beta_{2}$ AR-stimulated B cells (data not shown). It was not in the scope of this study to delineate, whether local suppressive effects occur in the joints, the lymph nodes, or the spleen. However, in adoptive transfer experiments, recipient animals were splenectomised before transfer of $B$ cells, which indicates that homing to the spleen is not necessary for the suppressive effect of B cells from SNS-intact animals.

As mentioned in the introduction, one problem with B-cell depleting strategies in rheumatic disease is the unselective approach, which depletes both, proinflammatory and antiinflammatory B cells. In light of our results, it might be possible to enrich anti-arthritic $B$ cells in vitro using $\beta_{2}$-adrenergic stimuli. Combining this approach with $\mathrm{B}$-cell depletion and retransfer of in vitro generated anti-arthritic B cells might open new therapeutic possibilities for the future.

Acknowledgements The authors thank Anja Sabo for excellent technical assistance.
Funding This study was funded by the institution and by the Deutsche Forschungsgemeinschaft (DFG FOR696, P0801/4-1).

Competing interests None.

Provenance and peer review Not commissioned; externally peer reviewed.

Contributors GP study design, data aquisition, statistical data analysis, data interpretation, manuscript preparation; MM study design, data aquisition; RHS study design, data interpretation, substantial manuscript revision.

\section{REFERENCES}

1. Mauri C, Gray D, Mushtaq N, et al. Prevention of arthritis by interleukin 10-producing B cells. J Exp Med 2003;197:489-501.

2. Mosser DM, Zhang X. Interleukin-10: new perspectives on an old cytokine. Immunol Rev 2008;226:205-18.

3. Yanaba $\mathbf{K}$, Bouaziz JD, Haas KM, et al. A regulatory B cell subset with a unique CD1dhiCD5 + phenotype controls T cell-dependent inflammatory responses. Immunity 2008;28:639-50.

4. Mauri C, Ehrenstein MR. The 'short' history of regulatory B cells. Trends Immunol 2008:29:34-40

5. Platzer C, Fritsch E, Elsner T, et al. Cyclic adenosine monophosphate-responsive elements are involved in the transcriptional activation of the human IL-10 gene in monocytic cells. Eur J Immunol 1999;29:3098-104.

6. Platzer C, Meisel C, Vogt K, et al. Up-regulation of monocytic IL-10 by tumor necrosis factor-alpha and cAMP elevating drugs. Int Immunol 1995;7:517-23.

7. Brenner S, Prösch S, Schenke-Layland K, et al. cAMP-induced Interleukin-10 promoter activation depends on CCAAT/enhancer-binding protein expression and monocytic differentiation. J Biol Chem 2003;278:5597-604.

8. Podojil JR, Sanders VM. CD86 and beta2-adrenergic receptor stimulation regulate B-cell activity cooperatively. Trends Immunol 2005;26:180-5.

9. Pongratz G, McAlees JW, Conrad DH, et al. The level of IgE produced by a B cell is regulated by norepinephrine in a p38 MAPK- and CD23-dependent manner. J Immunol 2006;177:2926-38.

10. Härle P, Möbius D, Carr DJ, et al. An opposing time-dependent immune-modulating effect of the sympathetic nervous system conferred by altering the cytokine profile in the local lymph nodes and spleen of mice with type II collagen-induced arthritis. Arthritis Rheum 2005;52:1305-13.

11. Härle $\mathbf{P}$, Pongratz $G$, Albrecht J, et al. An early sympathetic nervous system influence exacerbates collagen-induced arthritis via CD4+CD25+ cells. Arthritis Rheum 2008;58:2347-55.

12. Straub RH, Rauch L, Fassold A, et al. Neuronally released sympathetic neurotransmitters stimulate splenic interferon-gamma secretion from T cells in early type II collagen-induced arthritis. Arthritis Rheum 2008;58:3450-60.

13. Fassold A, Falk W, Anders S, et al. Soluble neuropilin-2, a nerve repellent receptor, is increased in rheumatoid arthritis synovium and aggravates sympathetic fiber repulsion and arthritis. Arthritis Rheum 2009;60:2892-901.

14. Heo YJ, Joo YB, Oh HJ, et al. IL-10 suppresses Th17 cells and promotes regulatory $T$ cells in the CD4 + T cell population of rheumatoid arthritis patients. Immunol Lett 2010;127:150-6.

15. Lombardi MS, Kavelaars A, Schedlowski M, et al. Decreased expression and activity of G-protein-coupled receptor kinases in peripheral blood mononuclear cells of patients with rheumatoid arthritis. FASEB J 1999;13:715-25.

16. Lombardi MS, Kavelaars A, Cobelens PM, et al. Adjuvant arthritis induces downregulation of $\mathrm{G}$ protein-coupled receptor kinases in the immune system. $\mathrm{J}$ Immunol 2001;166:1635-40.

17. de PB, Peri-Lopez M, Ballina-Garcia FJ, et al. Interleukin 10 and tumor necrosis factor-alpha genotypes in rheumatoid arthritis--association with clinical response to glucocorticoids. J Rheumatol 2010;37:503-11.

18. Marinou I, Healy J, Mewar D, et al. Association of interleukin-6 and interleukin-10 genotypes with radiographic damage in rheumatoid arthritis is dependent on autoantibody status. Arthritis Rheum 2007;56:2549-56.

19. Asadullah K, Sterry W, Volk HD. Interleukin-10 therapy-review of a new approach. Pharmacol Rev 2003;55:241-69.

20. Guichelaar T, ten Brink CB, van Kooten PJ, et al. Autoantigen-specific IL-10transduced $T$ cells suppress chronic arthritis by promoting the endogenous regulatory IL-10 response. J Immunol 2008;180:1373-81. 


\section{A RD The sympathetic nervous system stimulates anti-inflammatory B cells in collagen-type Il-induced arthritis}

Georg Pongratz, Madlen Melzer and Rainer H Straub

Ann Rheum Dis 2012 71: $432-439$ originally published online September 27, 2011

doi: 10.1136/ard.2011.153056

Updated information and services can be found at:

http://ard.bmj.com/content/71/3/432

These include:

References This article cites 19 articles, 7 of which you can access for free at: http://ard.bmj.com/content/71/3/432\#BIBL

Email alerting Receive free email alerts when new articles cite this article. Sign up in the service box at the top right corner of the online article.

Topic Articles on similar topics can be found in the following collections

Collections

Degenerative joint disease (4641)

Musculoskeletal syndromes (4951)

Biological agents (545)

Drugs: musculoskeletal and joint diseases (700)

Immunology (including allergy) (5144)

\section{Notes}

To request permissions go to:

http://group.bmj.com/group/rights-licensing/permissions

To order reprints go to:

http://journals.bmj.com/cgi/reprintform

To subscribe to BMJ go to:

http://group.bmj.com/subscribe/ 